\title{
Instituições de longa permanência para idosos e a covid-19: urgência no debate
}

Long stay institutions for the elderly and covid-19: emergency in the debate Instituciones de larga estancia para personas mayores y covid-19: emergencia en el debate

Recebido: 10/05/2020 | Revisado: 12/05/2020 | Aceito: 13/05/2020 | Publicado: 23/05/2020

\section{Adriana Valéria da Silva Freitas}

ORCID: https://orcid.org/0000-0003-1831-4537

Escola de Enfermagem da Universidade Federal da Bahia, Brasil

E-mail: adrianaf719@gmail.com

\section{Resumo}

Introdução: A COVID-19 é uma doença que tem como uma de suas características atingir mais agressivamente idosos e pessoas com doenças crônicas. Objetivo: apresentar uma breve reflexão sobre a urgência no debate em relação às Instituições de Longa Permanência para Idosos e a COVID-19. Metodologia: Estudo descritivo, reflexivo, com base em uma leitura crítica sobre a doença e seus possíveis impactos nas Instituições de Longa Permanência para Idosos. Para levantamento de dados, foi realizada uma pesquisa bibliográfica no Google Acadêmico sobre o tema. Resultados: As demandas trazidas pela infecção da COVID-19, apesar de exigirem métodos inovadores de intervenção, reafirmam a importância do Pacto da Saúde, em relação a atenção integral ao idoso. Propõe-se como uma das estratégias de enfrentamento para essa pandemia, pelas instituições de idosos, a construção de planos de contingência que considerem as especificidades desses espaços como um local de convivência coletiva e as diferentes demandas dos seus moradores. Conclusão: as reflexões acerca da urgência no debate sobre essas instituições como espaços vulneráveis a pandemia da COVID19, ponderaram sobre a diversidade existente nestes locais no que diz respeito aos residentes, estrutura física e capacidade dos profissionais em cuidar, com base nas especificidades do envelhecimento para evitar que sejam afetados por um drama que as marcaria como o epicentro da necropolítica.

Palavras-chave: Pandemia; COVID-19; Idoso; Instituição de longa permanência para idosos 


\section{Abstract}

Introduction: COVID-19 is a disease that has as one of its characteristics to reach more aggressively the elderly and people with chronic diseases. Objective: to present a brief reflection on the urgency of the debate in relation to Long Term Care Institutions for the Elderly and COVID-19. Methodology: Descriptive, reflective study, based on a critical reading about the disease and its possible impacts on Long Term Care Institutions for the Elderly. For data collection, a bibliographic search was carried out on Google Scholar on the subject. Results: The demands brought by the COVID-19 infection, despite requiring innovative methods of intervention, reaffirm the importance of the Health Pact, in relation to comprehensive care for the elderly. As one of the coping strategies for this pandemic by elderly institutions, it is proposed to build contingency plans that consider the specificities of these spaces as a place of collective coexistence and the different demands of its residents. Conclusion: reflections on the urgency in the debate about these instituicions as vulnerable spaces to the pandemic of COVID-19, pondered the diversity that exists in these places with regard to residents, physical structure and the capacity of professionals to care, based on the specificities of the aging to avoid being affected by a drama that would mark them as the epicenter of necropolitics.

Keywords: Pandemic; COVID-19; Elderly; Long term care facility for the elderly.

\section{Resumen}

Introducción: COVID-19 es una enfermedad que tiene como una de sus características alcanzar de manera más agresiva a los ancianos y personas con enfermedades crónicas. Objetivo: presentar una breve reflexión sobre la urgencia del debate en relación con las instituciones de atención a largo plazo para ancianos y COVID-19. Metodología: Estudio descriptivo, reflexivo, basado en una lectura crítica sobre la enfermedad y sus posibles impactos en las instituciones de atención a largo plazo para ancianos. Para la recolección de datos, se realizó una búsqueda bibliográfica en Google Scholar sobre el tema. Resultados: las demandas planteadas por la infección por COVID-19, a pesar de requerir métodos innovadores de intervención, reafirman la importancia del Pacto de Salud, en relación con la atención integral a los ancianos. Como una de las estrategias de afrontamiento de esta pandemia por parte de las instituciones de la tercera edad, se propone construir planes de contingencia que consideren las especificidades de estos espacios como un lugar de convivencia colectiva y las diferentes demandas de sus residentes. Conclusión: las reflexiones sobre la urgencia en el debate sobre estas instituciones como espacios vulnerables a la 
pandemia de COVID-19, reflexionaron sobre la diversidad existente en estos lugares con respecto a los residentes, la estructura física y la capacidad de los profesionales para atender, en función de las especificidades de la envejecimiento para evitar verse afectado por un drama que los marcaría como el epicentro de la necropolítica.

Palabras clave: Pandemia; COVID-19; Ancianos; Centro de atención a largo plazo para ancianos.

\section{Introdução}

A COVID-19 é uma doença que tem como uma de suas características atingir idosos, além das pessoas que possuem doenças crônicas, consideradas como um dos grupos mais suscetíveis a infecção (Belluz, 2020). Trata-se de uma doença de nível mundial e que, no Brasil, também é conhecida pelo seu agente causador, coronavírus. A população idosa ainda possui maiores condições de gravidade pelas pluripatologias, deficiências crônicas e disfunção no sistema imunológico que possam vir a apresentar (Bandaranayake, 2020).

Um estudo sobre essa doença, afirma que idosos residentes em Instituições de Longa Permanência (ILPI's), possuem maior suscetibilidade ao COVID-19, bem como os piores resultados dessa infecção (D’Adamo, Yoshicawa \& Ouslander, 2020).

Para que seja reduzida as chances de que as ILPI's sofram com as consequências da doença, será preciso agir o quanto antes. Prevendo o avanço da pandemia nas Instituições de Longa Permanência para Idosos, deve-se tomar como referências os casos ocorridos nos EUA, onde um surto de COVID-19, em uma casa de repouso na área de Seattle, Washington, foi associado a várias mortes, e relatórios mais recentes destacam a infecção em muitas outras ILPI's, com registro de óbitos nestes locais (Kamp \& Mathews, 2020).

Nesse contexto, a pandemia vem mostrando que o sistema de saúde brasileiro é frágil e sem condições de assumir a necessidade de cuidados de toda a população. Além disto, doenças crônicas como: cardíacas, cardiovasculares, pneumopatias entre outras, podem estar associadas a essa infecção, demonstrando que as condições de saúde das pessoas carecem de hábitos de vida saudáveis, não condizentes com a forma como a sociedade de consumo impõem comportamentos produtivistas sobre o cidadão.

Assim, as pessoas idosas que vivem em Instituições de Longa Permanência (ILPI's) não são frequentemente referenciadas. De uma maneira geral, são excluídas pela sua condição de vida e muitas vezes pelo abandono que vivenciam. Em relação à COVID-19 no Brasil, os noticiários e debates públicos pouco trazem sobre idosos institucionalizados. Dessa maneira, 
existem espaços mais propícios à disseminação do SARS-CoV-2, vírus de transmissão aérea, do que em ILPI's, onde seus moradores vivem confinados em estruturas precárias, pouco ventiladas, dividindo quartos e ainda não têm acesso a hábitos saudáveis que supram suas necessidades humanas básicas.

Diante do que foi aqui exposto, o objetivo deste estudo foi apresentar uma breve reflexão sobre a urgência no debate em relação às Instituições de Longa Permanência para Idosos e a doença causada pelo coronavírus.

\section{Metodologia}

Este estudo é descritivo, reflexivo como preconiza (Pereira, et al., 2018). Ele é construído com base na leitura crítica sobre a COVID-19 e suas possíveis repercussões nas Instituições de Longa Permanência para Idosos. Essa construção teórica aproxima-se da abordagem qualitativa, tendo em vista a interpretação e análise dos elementos teóricos obtidos por meio do levantamento bibliográfico, o qual apesar de não ter sido feito de maneira sistemática, por ser este um tema ainda com poucas publicações, foi realizado através de uma pesquisa exploratória de documentos, em formato eletrônico, disponíveis no Scholar Google sobre esta doença. Foram também utilizadas, outras fontes de informação, como os documentos do Ministério da Saúde e órgãos oficiais.

Após o levantamento bibliográfico, partiu-se para a leitura do material encontrado e análise de acordo com o objetivo traçado para este estudo, resultando em dois eixos de discussão: 1. Porque as ILPI's são espaços vulneráveis ao coronavírus? 2. Estratégias para as ILPI's no enfrentamento a COVID- 19. Estes eixos colaboram para a compreensão dos acontecimentos que são de relevância social.

\section{Resultados e Discussão}

\section{Porque as ILPI's são espaços vulneráveis ao coronavírus?}

As ILPI's são consideradas pela ANVISA como serviços governamentais ou não governamentais, de caráter residencial, destinada ao domicílio coletivo de pessoas que tenham idade igual ou superior a 60 anos, podendo possuir ou não suporte familiar, mas que lhe seja garantida a condição de liberdade, dignidade e cidadania (Anvisa, 2020) 
Contudo, não há um consenso sobre o que de fato é uma ILPI no Brasil, e isso a faz ser um espaço muito diverso, onde residem tanto idosos independentes, em relação à carência de renda e/ou de família, quanto àqueles com dificuldades para o desempenho das atividades básicas do dia a dia, que necessitem de cuidados prolongados (Camarano \& Kanso, 2010).

Essa diversidade faz com que seja preciso uma estrutura física que atenda as diferentes demandas dos idosos, um quadro de profissionais de saúde e cuidadores capacitados para cuidar das especificidades do envelhecimento, e ainda o apoio das famílias e da sociedade.

Dessa maneira, ao ser colocada frente a pandemia, as ILPI's precisam estar aparelhadas para cuidar de maneira preventiva, protegendo os idosos das sequelas que essa situação possa lhes causar. Porém, pela sua fragilidade enquanto espaço coletivo, neste cenário, deliberações da justiça que ampara aos idosos que residem em ILPI's são urgentes e importantes para reduzir as possibilidades de mortalidade desse grupo etário. A pandemia requer soluções efetivas, especialmente em países com baixa renda, condições desumanas e comportamentos que promovem o idadismo e, consequentemente, reforça o gerontocídio (Kalash, Ramos, Louvison, Veras \& Lima, 2020). Diante dessa situação, algumas estratégias são fundamentais para que as ILPI's possam passar pela pandemia cumprindo seu papel de cuidado as pessoas idosas.

\section{Estratégias para as ILPI's no enfrentamento a COVID- 19}

O distanciamento social é uma das medidas de prevenção que pode favorecer a contenção de modo efetivo em relação à infecção causada por essa enfermidade. Entretanto, há uma ampla discussão, pois de um lado, ele vem como promotor da saúde e de outro já é conhecido pelos idosos que foram abandonados pelos seus familiares e acabam sendo isolados pelo próprio processo de institucionalização. Frente a esse problema, vê-se a importância de que essas medidas de distanciamento social para pessoas idosas sejam discutidas no âmbito político e intersetorial com apoio da gerontologia, visando minimizar complicações como as síndromes geriátricas, sendo o isolamento social, uma delas (Hammerschmidt \& Santana, 2020).

O Pacto pela Saúde em um de seus três componentes que é o Pacto pela vida, refere a saúde do idoso como uma de suas prioridades. Dentre suas diretrizes está a atenção integral e integrada à saúde da pessoa idosa (Brasil, 2006a). No entanto, quando se fala em ILPI's, elas estão inseridas no campo de ação do Sistema Único de Assistência Social (SUAS), dispositivo de responsabilidade do Ministério do Desenvolvimento Social e Combate à Fome. Com 
isso, enfatiza-se a necessidade de ampliar essa compressão e amparar às ILPI'S de maneira integral: colocando-as também atreladas ao Sistema Único de Saúde (SUS).

Contudo, parte dos documentos sobre o combate a pandemia da COVID-19, no Brasil, menciona de maneira superficial, a população idosa e dentre elas as que estão institucionalizadas. Estando mais expressivas através de cartas de grupos de defesa das necessidades do idoso (Kalash, Ramos, Louvison, Veras, \& Lima, 2020) e notas técnicas (Brasil, 2020b). Sendo assim, uma das principais maneiras de prevenção da COVID-19 na população em geral, como o distanciamento social, torna-se de difícil aplicação nas ILPI's, pois os cuidadores que são os profissionais em maior contato com os idosos, muitas das vezes estão em vulnerabilidade pela condição de vida e trabalho, é o que defende Groisman (Fiocruz, 2020). Além disso, o cuidado dispensado, especialmente a idosos acamados, precisa de proximidade como condição de segurança e prevenção de acidentes e iatrogenias.

Dessa forma, a prevenção contra a COVID-19 não pode ser restringida ao impedimento de visitas de familiares e interrupção de ações de grupos voluntários, como aquelas, em que muitas instituições têm como forma de lazer para seus residentes. É necessário que seja construído um plano de contingência para as ILPI's, adequado as suas demandas e que permita a operacionalização das medidas preconizadas para a população geral, detalhando os procedimentos e as atribuições dos diferentes níveis de atenção para prevenir, detectar e confirmar os casos suspeitos da COVID-19, definindo a assistência e a vigilância epidemiológica.

Deixar de discutir e construir estratégias mais condizentes com o cotidiano das ILPI's, demonstra a exclusão da população idosa institucionalizada, das lacunas existentes nas políticas públicas estabelecidas para a mesma, contrariando os princípios do SUS com reflexos na assistência à saúde, na aquisição de equipamentos de proteção individual (EPI's) necessários para atuação no combate a COVID-19 nestes locais, assim como testes para diagnóstico. Sem contar com a não priorização das estratégias de vigilância epidemiológica, atitude que favorece a doença permanecer escondida por trás dos muros dessas instituições.

No contexto das ILPI's, a realização de inspeções para identificar prontamente a introdução do vírus nestes locais e, o imediato bloqueio de transmissão, são indispensáveis para evitar a rápida disseminação. Com isso, a quarentena decretada no país, deve chegar as ILPI's de maneira mais rápida para o controle da transmissão, desde que os idosos residentes assintomáticos sejam mantidos separados daqueles sintomáticos. Como muitas dessas instituições não possuem estruturas que permitam o isolamento em um quarto individual, é 
preconizado o isolamento de coorte, ou seja, respeitando uma distância mínima superior a 1 metro entre os leitos. (Brasil, 2020c).

É importante realizar o teste do tipo RT-PCR para todas as pessoas que apresentem sintomas compatíveis com a COVID-19, mesmo que sejam leves, devem ser testados imediatamente e, se positivos, o isolamento é a orientação a ser dada. A vacinação contra gripe é uma medida importante porque poderá reduzir, a ocorrência de sintomas gripais por outros vírus respiratórios, reduzindo o número de pessoas que precisarão do teste para a COVID-19. (Brasil, 2020c)

Dessa forma, a testagem das pessoas idosas em ILPI's, cuidadores e profissionais de saúde com quadro gripal deveriam ser considerados estratégia prioritária para o enfrentamento da pandemia nestes espaços. Contudo, por enquanto, as ILPI's não se encontram como locais prioritários para a testagem dos casos suspeitos. Assim, a não divulgação desses casos, dos confirmados ou de óbitos por coronavírus em idosos residentes em ILPI's, pode ser contestada diante da não realização do teste diagnóstico.

O cuidado dos casos suspeitos também pode ser considerado como outro aspecto de difícil controle. Em relação a população em geral, todo paciente com sintomas de resfriado comum ou quadro de gripe recomenda-se ser tratado como possível infecção pelo novo coronavírus, seguindo as orientações do Ministério da Saúde. Para os casos classificados como leves a indicação é o isolamento por 14 dias após o início dos sintomas, e para os casos graves devem ser encaminhados pelo sistema de regulação de urgências (Brasil, 2020c).

Nas ILPI's, perceber o risco à vida e à saúde que a COVID-19 provoca, somada ao isolamento dos idosos nestes locais, à interrupção das atividades de visitas, de lazer e até mesmo às religiosas são fatores de estresse, com fortes implicações emocionais para os idosos. Alguns estudos referem a necessidade de atentar para sintomas depressivos em idosos (Pimentel, Rodrigues \& Silva, 2020). Essa ocorrência pode ter relação com a suspensão do contato com a família que favorece a intensificação da sensação de isolamento, insegurança e solidão, gerando preocupação com a saúde dos familiares. Além de preocupações com sua própria vida e o medo da morte e abandono.

Para reduzir o sentimento de perda de controle e ansiedade proveniente da situação vivenciada, é preciso que as ILPI's sejam constantemente monitoradas e informadas sobre possíveis estratégias para proteger, prevenir e cuidar da saúde dos residentes, visando manter a comunicação com seus familiares por cartas, telefones, vídeos e outras maneiras disponibilizadas pela instituição. É importante evitar, ainda, a estigmatização, os abusos e 
violências que podem ocorrer contra a pessoa idosa, identificada como possível portadora do vírus.

Nessa conjuntura, prover os profissionais de saúde e cuidadores com informações, disponibilizar EPI's, testes diagnóstico, vacina contra a gripe e orientações para ajuste das práticas de cuidado frente ao risco da doença, são fundamentais, além do afastamento do trabalho dos que pertencem ao grupo de risco. Enfatiza-se o papel dos órgãos de fiscalização, Ministério Público e a Defensoria Pública. Assim como o Conselho do idoso, para conhecer a verdadeira situação epidemiológica das ILPI's, assegurando a implantação das medidas preconizadas.

Cabe ressaltar também que de acordo com a Organização Mundial da Saúde (OMS), todas as decisões clínicas devem ser tomadas pela equipe de saúde, não devendo ser ignoradas por outros trabalhadores das ILPI's. A situação de emergência da COVID-19 não pode ser uma justificativa para impedir inspeções nestes locais. Contudo, vale destacar que o bloqueio total das ILPI's, com isolamento coletivo dos idosos e a limitação de vistas de familiares e outras pessoas não são suficientes para evitar a disseminação do vírus.

\section{Considerações Finais}

Este estudo discorreu sobre as ILPI's como espaços vulneráveis a pandemia da COVID-19. As reflexões acerca da urgência no debate ponderaram sobre essas instituições e sua diversidade no que diz respeito aos residentes, estrutura física e capacidade dos profissionais em cuidar, com base nas especificidades do envelhecimento.

A necessidade de ter estratégias condizentes com as ILPI's deve ser considerada como fundamental para amparar pessoas idosas, residentes nestes locais, e minorar os efeitos da doença.

A efetiva implantação, de maneira transparente e ética, de estratégias de combate e vigilância, fundamentadas pelo conhecimento científico, são necessárias e indispensáveis para evitar que as ILPI's possam ser cenários de um drama que as marcaria como o epicentro da necropolítica.

Diante das reflexões construídas, recomendam-se estudos que possam caracterizar as ILPI's, colaborando para que seja possível conhecer esses espaços e melhor contribuir para que suas necessidades sejam supridas, fortalecendo-os para o enfrentamento de situações emergenciais como a pandemia da COVID-19. 


\section{Referências}

Anvisa (2005). Resolução da Diretoria Colegiada, 283, de 26 de setembro de 2005. Acesso em: 08 de maio de 2020. Disponível em:

http://portal.anvisa.gov.br/documents/10181/2718376/RDC_283_2005_COMP.pdf/a38f2055c23a-4eca-94ed-76fa43acb1pdf

Bandaranayake, T., \& Shaw, AC. (2016). Host resistance and immune aging. Clin Geriatr Med., 32(1): 415-32. Acesso em: 03 de maio de 2020. Disponível em: https://www.ncbi.nlm.nih.gov/pmc/articles/PMC6986475/

Belluz, J. (2020, março 3). China's cases of Covid-19 are finally declining. A WHO expert explains why. Acesso em: 03 de maio de 2020. Disponível em: https://www.vox.com/2020/3/2/21161067/coronavirus-covid19-china

Brasil, MS. (2006a). Portaria no 399. Divulga o Pacto pela Saúde 2006 - Consolidação do SUS e aprova as Diretrizes Operacionais do Referido Pacto. Diário Oficial da União, 22 fev. 2006 Acesso em: 09 de maio de 2020. Disponível em:

https://bvsms.saude.gov.br/bvs/saudelegis/gm/2006/prt0399_22_02_2006.html

Brasil, MS. (2020b). Nota Técnica n 8/2020-COSAPI/CGCIVI/DAPES/SAPS/MS.

Prevenção e controle pelo novo coronavírus (SARS-Cov-2) a serem adotadas nas Instituições de Longa Permanência para Idosos. Acesso em: 08 de abril de 2020. Disponível em: http://189.28.128.100/dab/docs/portaldab/documentos/notatecnica82020COSAPICGCIVIDA PESSAPSMS02abr2020COVID-19.pdf

Brasil, MS. (2020c). Nota Técnica GVIMS/GGTES/ANVISA Nº4/2020. Orientações para serviços de saúde: medidas de prevenção e controle que devem ser adotadas durante a assistência aos casos suspeitos ou confirmados de infecção pelo novo coronavírus (SARSCoV2). Acesso em: 03 de maio de 2020. Disponível em: http://portal.anvisa.gov.br/documents/33852/271858/Nota+T\%C3\%A9cnica+n+042020+GVIMS-GGTES-ANVISA/ab598660-3de4-4f14-8e6f-b9341c196b28 
Camarano, AA, \& Kanso, S. (2010). As instituições de longa permanência para idosos no Brasil. Revista Brasileira de Estudos de População, 27(1): 233-5. Acesso em: 03 de maio de 2020. Disponível em: https://www.scielo.br/pdf/rbepop/v27n1/14.pdf

D’Adamo, H, Yoshikawa, T \& Ouslander, JG. (2020, março 25). Coronavirus disease 2019 in geriatrics and long-term care: the ABCDs of COVID-19. Jornal da Sociedade Americana de de Geriatria. Acesso em: 08 de maio de 2020. Disponível: https://doi.org/10.1111/jgs.16445

Fiocruz. (2020, março 23). As cuidadoras de idosos estão expostas também a uma situação de risco, já que o cuidado é um trabalho de proximidade. Acesso em: 29 de abril de 2020. Disponível em: http://www.epsjv.fiocruz.br/noticias/entrevista/cuidadoras-de-idosos-estaoexpostas-tambem-a-uma-situacao-de-risco-ja-que-o

Hammerschmidt, KSA \& Santana, RF. (2020). Saúde do idoso em tempos de pandemia COVID-19. Cogitare Enfermagem, 25: (e72849), 1-10. Acesso em: 08 de maio de 2020. Disponível em: http://dx.doi.org/10.5380/ce.v25i0.72849.

Kalash AS, Ramos, Luiz., Louvison, M, Veras, R \& Lima, K. (2020, março 27). Carta Aberta ao Conselho Nacional dos Direitos da Pessoa Idosa - CNDI. Acesso em: 28 de abril de 2020. Disponível em: http://ilcbrazil.org/portugues/noticias/carta-aberta-ao-conselho-nacional-dosdireitos-da-pessoa-idosa-cndi/

Kamp J \& Mathews AW. (2020, março 19). Coronavirus outbreaks spreading in nursing homes. Wall Street Journal. Acesso em: 03 de maio de 2020. Disponível em: https://www.wsj.com/articles/coronavirus-outbreaks-spreading-in-nursing-homes11584628291.

Pereira, AS, Shitsuka, DM, Parreira, FJ \& Shitsuka, R. (2018). Metodologia da pesquisa científica. [e-book]. Santa Maria. Ed. UAB/NTE/UFSM. Acesso em: 21 maio de 2020. Disponível em: https://repositorio.ufsm.br/bitstream/handle/1/15824/Lic_Computacao_MetodologiaPesquisa-Cientifica.pdf? sequence $=1$ 
Pimentel, ASG, Rodrigues, MN, \& Silva, M.O. (2020) Saúde psíquica em tempos de

Coronavírus. Research, Society and Development, 9(7). Acesso em: 08 de maio de 2020.

Disponível em: https://rsd.unifei.edu.br/index.php/rsd/article/viewFile/3602/2807

\section{Porcentagem de contribuição de cada autor no manuscrito}

Adriana Valéria da Silva Freitas - 100\% 